FLUID DYNAMICS

\section{To merge or not to merge ...}

Frieder Mugele

\section{... that is the dilemma addressed in a study of oppositely charged liquid drops controlled by an electric field. Contrary to conventional wisdom, beyond a critical charge, the drops fail to merge.}

Just as we may be guided by a sense of 'more is more' in our daily lives, so physicists often use this principle as a good first guess to explain physical phenomena. For example, in a situation as simple as two objects with electrically opposite charges, the expectation is that the more strongly charged the objects are, the more they should attract each other. In the case of liquid drops, we would expect them to bump into each other and coalesce into one larger drop, and that, the higher their charge, the quicker this would happen. It seems that the simpler a situation is, the more stunned we are if nature 'refuses' to follow our expectations. Such a refusal is exactly what Ristenpart et al. ${ }^{1}$ find in experiments described on page 377 of this issue. Counter-intuitively, the authors show that two oppositely charged drops bounce off each other and do not coalesce if their charge exceeds a threshold value.

Ristenpart and colleagues' experiment ${ }^{1}$ is simple. A millimetre-sized water drop immersed in a beaker filled with oil becomes positively charged when brought into contact with a metal wire at high voltage. A second, much bigger drop, covering the bottom of the beaker, is kept at ground voltage and becomes negatively charged. The resulting electric field pulls the small drop towards the big one. For low to moderate electric-field strengths, or equivalently for low drop charges, the small drop is attracted towards the large one, deforms, touches and coalesces with it. For high field strengths, and beyond a critical value, the droplet is still attracted towards the larger drop and deforms on approaching it, but instead of coalescing, it bounces off and moves away towards the wire.

The authors ${ }^{1}$ show that this phenomenon is independent of the fluids involved in the process: the non-coalescence behaviour occurs for clean water drops in silicone oil, brine drops in crude oil, vinegar drops in olive oil, and water drops in air. Under suitable conditions, the small drop can even shuttle back and forth several times between the wire and the big drop. This effect is similar to the to-and-fro motion that small scraps of paper or Styrofoam experience when placed between the two electrodes of a high-voltage generator, and is usually demonstrated in first-year undergraduate laboratory experiments on electrostatics.

To understand the non-coalescence of oppositely charged drops, Ristenpart et al. used high-speed video microscopy to record drop motion right at the moment of coalescence/ non-coalescence. By doing so, they found that all drops, irrespective of their charge, established contact by forming a microscopic capillary bridge. But whereas for low-charge drops the bridge remained stable and sucked the small drop into the big one, for high-charge drops it broke up in less than a millisecond.

One of the beauties of Ristenpart and colleagues' work ${ }^{1}$ is that it provides a simple and convincing explanation for the observed phenomenon. As long as the drops are apart, electrostatic forces pull their surfaces towards each other, producing conically shaped protrusions a

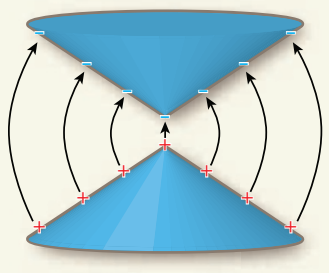

b

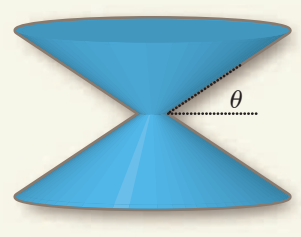

$\theta<\theta_{\mathrm{c}}$

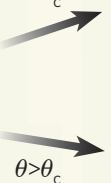

$\theta>\theta_{\mathrm{c}}$

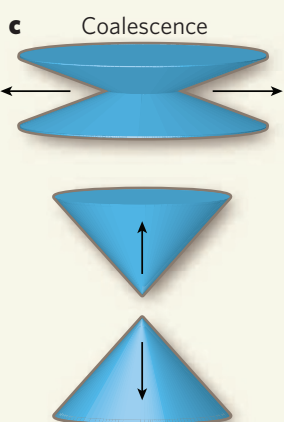

Non-coalescence

Figure 1 | Geometry of liquid-drop motion. a, The leading edges of two approaching liquid drops of opposite charge elongate and acquire a conical shape just before contact; the black lines represent the electric field created by the charged drops. b, On contact, the intersecting cones are characterized by a cone angle, $\theta$, that depends on the strength of the electric field just before contact. $c$, Ristenpart and colleagues ${ }^{1}$ show that the fate of the drops is determined by the cone angle at contact: below a critical cone angle, $\theta_{c}$, the drops coalesce; above $\theta_{c}$, the drops fail to coalesce.

known as Taylor cones ${ }^{2}$ at the drops' leading edges. But as soon as the capillary bridge is formed, the two drops are immediately electrically short-circuited and hence all electrostatic forces vanish abruptly. From that moment on, the surface tension of the drops in combination with the geometry of the Taylor cones determines the fate of the capillary bridge. For low-charge drops, the cones are blunt and hydrodynamically stable. They grow and suck the small drop into the big one. For high-charge drops, the cones are sharper and unstable. As a result, they break and the drops bounce off each other. The coalescence/ non-coalescence phenomenon is thus a consequence of the geometry of the Taylor cones initially formed under the influence of the electric field and the subsequent sudden vanishing of the electrostatic forces (Fig. 1).

Interestingly, Ristenpart et al. find that the threshold charge for non-coalescence increases for very low salt concentrations in water - that is, for poorly conducting water. Such conductivity dependence has also been observed in other electrohydrodynamic phenomena, including the emission of jets and drops from Taylor cones ${ }^{3}$ and the periodic detachment and reattachment of sessile liquid drops from a wire through the application of a voltage ${ }^{4}$. The latter experiment ${ }^{4}$ explains the conductivity dependence in terms of the increasing electrical resistivity during the break-up of the capillary bridge, and might well help to explain Ristenpart and co-workers' observations at low salt concentrations.

What will be the impact of Ristenpart and colleagues' work ${ }^{1}$ ? Their observation of the counter-intuitive, non-coalescing behaviour of charged liquid drops doesn't have an immediate link to a specific scientific problem or technological application. However, given the general validity of the phenomenon for any liquid-liquid or liquid-gas system, their study will provide insights into our understanding of a variety of well-known physical problems that involve drops in electric fields. For example, it should further our understanding of electrocoalescence-based de-emulsification processes that are widely used to separate residual water from crude oil. Moreover, it should help to explain the long-standing problem of cloud electrification in thunderstorms, which was addressed by Lord Rayleigh ${ }^{5}$ and Geoffrey Taylor $^{2}$ in their seminal contributions to the field of electrohydrodynamics.

Frieder Mugele is at the MESA+ Institute for Nanotechnology and the Faculty of Science and Technology, University of Twente, 7500 AE Enschede, the Netherlands. e-mail: f.mugele@utwente.nl

Ristenpart, W. D. Bird, J. C. Belmonte, A., Dollar, E. \& Stone, H. A. Nature 461, 377-380 (2009)

2. Taylor, G. Proc. R. Soc. Lond. A 280, 383-397 (1964).

3. Collins, R. T., Jones, J. J., Harris, M. T. \& Basaran, O. A Nature Phys. 4, 149-154 (2008)

4. Baret, J.-C. \& Mugele, F. Phys. Rev. Lett. 96, 016106 (2006).

5. Lord Rayleigh Proc. R. Soc. Lond. 28, 405-409 (1879). 